\title{
ON RELAXATION NORMALITY IN THE FUGLEDE-PUTNAM THEOREM FOR A QUASI-CLASS $A$ OPERATORS
}

\author{
M. H. M. RASHID AND M. S. M. NOORANI
}

\begin{abstract}
Let $T$ be a bounded linear operator acting on a complex Hilbert space $\mathcal{H}$. In this paper, we show that if $A$ is quasi-class $A, B^{*}$ is invertible quasi-class $A, X$ is a Hilbert-Schmidt operator, $A X=X B$ and $\left\|\left|A^{*}\right|\right\|\left|\left\||B|^{-1}\right\| \leq 1\right.$, then $A^{*} X=X B^{*}$.
\end{abstract}

\section{Introduction}

Let $\mathcal{H}$ be a complex Hilbert space, and let $\mathbf{B}(\mathcal{H})$ denote the algebra of all bounded linear operators on $\mathcal{H}$. If $T \in \mathbf{B}(\mathcal{H})$, we shall write $\operatorname{ker}(T), \operatorname{ran}(T)$ for the null space and range of $T$, respectively. An operator $T$ is said to be positive (denoted by $T \geq 0$ ) if $\langle T x, x\rangle \geq 0$ for all $x \in \mathcal{H}$ and also $T$ is said to be strictly positive (denoted by $T>0$ ) if $T$ is positive and invertible.

Recall $([1,10,12])$ that an operator $T$ is called p-quasihyponormal if $T^{*}\left(\left(T^{*} T\right)^{p}-\right.$ $\left.\left(T T^{*}\right)^{p} T\right) \geq 0$ for $p \in(0,1]$, and $T$ is called paranormal if $\left\|T^{2} x\right\| \geq\|T x\|^{2}$ for all unit vector $x \in \mathcal{H}$. Following $[7,9,13]$ we say that $T \in \mathbf{B}(\mathcal{H})$ belongs to class $A$ if $\left|T^{2}\right| \geq|T|^{2}$ and $T$ is called normaloid if $\left\|T^{n}\right\|=\|T\|^{n}$, for $n \in \mathbb{N}$ (equivalently, $\|T\|=r(T)$, the spectral radius of $T)$. Recall [2], an operator $T \in \mathbf{B}(\mathcal{H})$ is said to be $\omega$-hyponormal if $|\widetilde{T}| \geq|T| \geq\left|\widetilde{T^{*}}\right|$. We remark that $\omega$-hyponormal operator is defined by using Aluthge transformation $\widetilde{T}=|T|^{\frac{1}{2}} U|T|^{\frac{1}{2}}$. An operator $T$ is said to be quasi-class $A$ if

$$
T^{*}\left|T^{2}\right| T \geq T^{*}|T|^{2} T \text {. }
$$

The quasi-class $A$ operators were introduced, and their properties were studied in [11]. (see also [6]). In particular, it was shown in [11] that the class of quasi-class $A$ operators contains properly classes of class $A$ and p-quasihyponormal operators. quasiclass $A$ operators were independently introduced by Jeon and Kim [11]. They gave an example of a quasi-class $A$ operator which is not paranormal nor normaloid. Jeon and Kim example show that neither the class paranormal operators nor the class of quasiclass $A$ contains the other. We shall denote classes of $\omega$-hyponormal, $p$-quasihyponormal operators, paranormal operators, normaloid operators, class $A$ operators, and quasi-class A operators by $\omega \mathcal{H}, \mathcal{Q H}(p), \mathcal{P N}, \mathcal{N}, \mathcal{A}$, and $\mathcal{Q} \mathcal{A}$, respectively. It is well known that

$$
\omega \mathcal{H} \subset \mathcal{A} \subset \mathcal{P N} \subset \mathcal{N} \quad \text { and } \quad \mathcal{Q H}(p) \subset \mathcal{P N} \subset \mathcal{N},
$$

Received May 20, 2008; revised December 8, 2008.

2000 Mathematics Subject Classification. 47A10, 47B20.

Key words and phrases. Fuglede-Putnam theorem, quasi-class $A$. 
also, the following inclusions holds;

$$
\mathcal{A} \subset \mathcal{Q A} \quad \text { and } \quad \mathcal{Q H}(p) \subset \mathcal{Q A} \text {. }
$$

\section{Properties of quasi-class $A$ operators}

We begin this section by the following famous lemma.

Lemma 2.1.([11]) Let $T \in \mathbf{B}(\mathcal{H})$. Suppose that $T$ is quasi-class $A$ with not have dense range. Then

$$
T=\left(\begin{array}{cc}
B & S \\
0 & 0
\end{array}\right) \quad \text { on } \quad \mathcal{H}=\overline{\operatorname{ran}(T)} \oplus \operatorname{ker}\left(T^{*}\right),
$$

where $B=\left.T\right|_{\operatorname{ran}(T)}$ is the restriction of $T$ to $\overline{\operatorname{ran}(T)}$, and $B$ is belongs to class $A$. Moreover, $\sigma(T)=\sigma(B) \cup\{0\}$.

It is a slight generalization of [1, Corollary 3] we have

Theorem 2.2. If $T \in \mathbf{B}(\mathcal{H})$ is a quasi-class $A$ and $T^{*}$ is $\omega$-hyponormal, then $T$ is normal.

Proof. Since $T^{*}$ is $\omega$-hyponormal, it follows from [1, Theorem 4] that $\left|T^{* 2}\right| \geq\left|T^{*}\right|^{2}$ and $\left|T^{2}\right| \leq|T|^{2}$. Hence $T^{*}\left|T^{2}\right| T=T^{*}|T|^{2} T$ because $T$ is quasi-class $A$. Now $T^{*}|T|^{2} T=$ $T^{* 2} T^{2}=|T|^{4}=\left(T^{*} T\right)^{2}$ and $P\left(T^{*} T-T T^{*}\right) P=0$, where $P$ is the orthogonal projection onto $\overline{\operatorname{ran}(T)}$. Let

$$
T=\left(\begin{array}{cc}
B & S \\
0 & 0
\end{array}\right) \quad \text { on } \quad \mathcal{H}=\overline{\operatorname{ran}(T)} \oplus \operatorname{ker}\left(T^{*}\right)
$$

be the matrix representation of $T$. Then $P\left(T^{*} T-T T^{*}\right) P=0$ implies that $B^{*} B=$ $B B^{*}+S S^{*}$. Since $T^{*}$ is $\omega$-hyponormal, then $T^{*}$ belongs to class $A$, so we have

$$
\begin{aligned}
B^{*} B=B B^{*}+S S^{*} & =P T T^{*} P \leq P\left|T^{* 2}\right| P=\left(B^{2} B^{* 2}+B S S^{*} B^{*}\right)^{\frac{1}{2}} \\
& =\left[B\left(B B^{*}+S S^{*}\right) B^{*}\right]^{\frac{1}{2}} \\
& =B B^{*}
\end{aligned}
$$

Hence $S=0$ and $B$ is normal. Therefore $T$ is normal.

\section{Generalized Fuglede-Putnam Theorem}

Let $\mathcal{C}_{2}$ denote the Hilbert-Schmidt class. Let $T \in \mathcal{C}_{2}$. Suppose $\left\{e_{n}\right\}$ is an orthonormal basis for $\mathcal{H}$. We define the Hilbert-Schmidt norm of $T$ to be

$$
\|T\|_{2}=\left(\sum_{n=1}^{\infty}\left\|T e_{n}\right\|^{2}\right)^{\frac{1}{2}} \text {. }
$$


This definition is independent of the choice of basis (see[5]). If $\|T\|_{2}<\infty, T$ is said to be a Hilbert-Schmidt operator.

Let $\mathcal{C}_{1}$ be the set $\left\{C=A B \mid A, B \in \mathcal{C}_{2}\right\}$. Then operators belonging to $\mathcal{C}_{1}$ are called trace class operators.

We define a linear functional

$$
\operatorname{tr}: \mathcal{C}_{1} \rightarrow \mathbb{C} \text { by } \operatorname{tr}(T)=\sum_{n=1}^{\infty}\left\langle C e_{n}, e_{n}\right\rangle
$$

for an orthonormal basis $\left\{e_{n}\right\}$ for $\mathcal{H}$.

In this case, the definition of $\operatorname{tr}(C)$ does not depend on the choice of an orthonormal basis and $\operatorname{tr}(C)$ is called the trace of $C$.

Theorem 3.1.([5]) We have the following properties.

(a) The set $\mathcal{C}_{2}$ is self adjoint ideal of $\mathbf{B}(\mathcal{H})$.

(b) If $\langle A, B\rangle=\sum_{n=1}^{\infty}\left\langle A e_{n}, B e_{n}\right\rangle=\operatorname{tr}\left(B^{*} A\right)=\operatorname{tr}\left(A B^{*}\right)$ for $A$ and $B$ in $\mathcal{C}_{2}$ and for any orthonormal basis $\left\{e_{n}\right\}$ for $\mathcal{H}$, then $\langle.,$.$\rangle is an inner product on \mathcal{C}_{2}$ and $\mathcal{C}_{2}$ is a Hilbert-Schmidt space with respect to this inner product.

Theorem 3.2.([5])If $T \in \mathbf{B}(\mathcal{H})$ and $A \in \mathcal{C}_{2}$, then

(i) $\|A\| \leq\|A\|_{2}$,

(ii) $\|T A\|_{2} \leq\|T\|\|A\|_{2}$,

(iii) $\|A T\|_{2} \leq\|A\|_{2}\|T\|$.

For each pair of operators $A, B \in \mathbf{B}(\mathcal{H})$, there is an operator $\Gamma$ defined on $\mathcal{C}_{2}$ via the formula $\Gamma X=A X B$, which due to [3]. Evidently, by Theorem 3.1 and Theorem 3.2, $\|\Gamma\| \leq\|A\|\|B\|$ and the adjoint of $\Gamma$ is given by the formula $\Gamma^{*} X=A^{*} X B^{*}$, its easily to see that from the calculation $\left\langle\Gamma^{*} X, Y\right\rangle=\langle X, \Gamma Y\rangle=\langle X, A Y B\rangle=\operatorname{tr}\left((A Y B)^{*} X\right)=$ $\operatorname{tr}\left(X B^{*} Y^{*} A^{*}\right)=\operatorname{tr}\left(A^{*} X B^{*} Y^{*}\right)=\left\langle A^{*} X B^{*}, Y\right\rangle$. If $A \geq 0$ and $B \geq 0$, then also $\Gamma \geq 0$ and $\Gamma^{\frac{1}{2}} X=A^{\frac{1}{2}} X B^{\frac{1}{2}}$ because of

$$
\begin{aligned}
\langle A X, X\rangle & =\operatorname{tr}\left(A X B X^{*}\right)=\operatorname{tr}\left(A^{\frac{1}{2}} X B X^{*} A^{\frac{1}{2}}\right) \\
& =\operatorname{tr}\left(\left(A^{\frac{1}{2}} X B^{\frac{1}{2}}\right)\left(A^{\frac{1}{2}} X B^{\frac{1}{2}}\right)^{*}\right) \geq 0 .
\end{aligned}
$$

The classical Fuglede-Putnam theorem asserts that if $A, B, X \in \mathbf{B}(\mathcal{H})$ such that $A X=$ $X B$, and if $A$ and $B$ are normal, then also $A^{*} X=X B^{*}$ (see [8, Problem 192]).

Theorem 3.3. Let $A, B^{*}, X \in \mathbf{B}(\mathcal{H})$. Suppose that $A$ is quasi-class $A, B^{*}$ is invertible quasi-class $A$ and $X$ is a Hilbert-Schmidt operator. Assume that $A X=X B$, then the operator $\Gamma$ defined by $\Gamma X=A X B$ is a quasi-class $A$ operator. 
Proof. Since $A$ and $B^{*}$ are quasi-class $A$, we have

$$
\begin{aligned}
\left(\Gamma^{*}\left|\Gamma^{2}\right| \Gamma-\Gamma^{*}|\Gamma|^{2} \Gamma\right) X & =A^{*}\left|A^{2}\right| A X B\left|B^{* 2}\right| B^{*}-A^{*}|A|^{2} A X B\left|B^{*}\right|^{2} B^{*} \\
& =A^{*}\left(\left|A^{2}\right|-|A|^{2}\right) A X B\left|B^{* 2}\right| B^{*} \\
& +A^{*}|A|^{2} A X\left(B\left|B^{* 2}\right| B^{*}-B\left|B^{*}\right|^{2} B^{*}\right) \\
& \geq 0
\end{aligned}
$$

this show that $\Gamma$ is quasi-class $A$.

Lemma 3.4. (Hölder-McCarthy Inequality) Let $B$ be a positive operator. Then the following inequalities hold for all $x \in \mathcal{H}$;

(1) $\left\langle B^{\alpha} x, x\right\rangle \leq\langle B x, x\rangle^{\alpha}\|x\|^{2(1-\alpha)}$, for $0<\alpha \leq 1$.

(2) $\left\langle B^{\alpha} x, x\right\rangle \geq\langle B x, x\rangle\|x\|^{2(1-\alpha)}$ for $\alpha \geq 1$.

Lemma 3.5. Let $B \in \mathbf{B}(\mathcal{H})$. If $B^{*}$ is quasi-class $A$ and invertible, then $\left(B^{*}\right)^{-1}$ is quasi-class $A$.

Proof. We cite the following obvious result (see [9]): Let $S$ be an invertible operator. Then

$$
\left(S^{*} S\right)^{\lambda}=S^{*}\left(S S^{*}\right)^{\lambda-1} S \quad \text { holds for any real number } \lambda .
$$

Suppose that $B^{*}$ is quasi-class $A$ and invertible. Then

$$
\begin{aligned}
B B B^{*} B^{*}=B\left|B^{*}\right|^{2} B^{*} & \leq B\left|B^{* 2}\right| B^{*} \quad\left(B^{*} \text { is quasi-class } A\right) \\
& =B\left(B^{2} B^{* 2}\right)^{\frac{1}{2}} B^{*} \\
& =B^{3}\left(B^{* 2} B^{2}\right)^{\frac{-1}{2}} B^{* 3} \quad \text { (by Equation 3.1) }
\end{aligned}
$$

(3.2) holds if and only if

$$
B^{-1} B^{*-1} \leq\left(B^{* 2} B^{2}\right)^{\frac{-1}{2}}=\left(B^{-2} B^{*-2}\right)^{\frac{1}{2}}=\left|B^{*-2}\right|
$$

Hence,

$$
\left|B^{*-1}\right|^{2} \leq\left|B^{*-2}\right| \text { and so } B^{-1}\left|B^{*-1}\right|^{2} B^{*-1} \leq B^{-1}\left|B^{*-2}\right| B^{*-1} \text {. This end the proof. }
$$

Theorem 3.6. Let $A, B, X \in \mathbf{B}(\mathcal{H})$. If $A$ is quasi-class $A, B^{*}$ is invertible quasi-class $A$ and $X$ is a Hilbert-Schmidt operator. Suppose that $A X=X B$ and $\left\|\left|A^{*}\right|\right\|\left\|\left.|| B\right|^{-1}\right\| \leq 1$, then $A^{*} X=X B^{*}$.

Proof. Let $\Gamma$ be a Hilbert-Schmidt operator defined by $\Gamma X=A X B^{-1}$ for all $X \in \mathcal{C}_{2}$. Since $\left(B^{*}\right)^{-1}=\left(B^{-1}\right)^{*}$ is quasi-class $A$ then Theorem 3.3 implies $\Gamma$ is quasi-class $A$. Since $\Gamma X=X$ and since $\Gamma$ is quasi-class $A$, we have

$$
\left\langle\left|\Gamma^{2}\right| X, X\right\rangle \geq\left\langle|\Gamma|^{2} X, X\right\rangle,
$$


by Hölder-McCarthy inequality, we have

$$
\begin{array}{rlr}
\||\Gamma| X\|^{2} & =\left\langle|\Gamma|^{2} X, X\right\rangle & \\
& \leq\left\langle\left(\Gamma^{* 2} \Gamma^{2}\right)^{\frac{1}{2}} X, X\right\rangle & \text { (by Equation 3.3) } \\
& \leq\|X\|\left\langle\Gamma^{* 2} \Gamma^{2} X, X\right\rangle^{\frac{1}{2}} & \text { (by Hölder McCarthy inequality) } \\
& =\|X\|\left\langle\Gamma^{2} X, \Gamma^{2} X\right\rangle^{\frac{1}{2}} & \\
& =\|X\|^{2},
\end{array}
$$

and hence

$$
\begin{aligned}
\left\|\Gamma^{*} X\right\| & =\left\|\left|\Gamma^{*}\right| X\right\| \\
& \leq\left\|\left|A^{*}\right|\right\|\left\||B|^{-1}\right\|\|X\| \\
& \leq\|X\| .
\end{aligned}
$$

Thus $\left\|\Gamma^{*} X-X\right\|^{2} \leq\left\|\Gamma^{*} X\right\|^{2}-2\|X\|^{2}+\|X\|^{2} \leq 0$. So, $A^{*} X\left(B^{*}\right)^{-1}=X$ which ends the proof.

\section{Acknowledgements}

The authors would like to thank the referee for his valuable suggestions for improving the original manuscript.

\section{References}

[1] A. Aluthge and D. Wang, An operator inequality which implies paranormality, Math. Ineq. Appl., 2(1999), 113-119.

[2] A. Aluthge and D. Wang, w-hyponormal operators, Integral Equations Operator Theory, 36(2000), 1-10.

[3] S. K. Berberian, An extension of a theorem of Fuglede-Putnam, Proc. Amer. Math. Soc., 71(1978), 113-114.

[4] M. Chō and T. Yamazaki, An operator transform from class A to the class of hyponormal operators and its application, Integral Equations Operator Theory, 53(2005), 497-508.

[5] J. B. Conway, A Course in Functional Analysis, Second Edition, Springer-Verlag, New york, 1990.

[6] B. P. Duggal, I. H. Jeon and I. H. Kim, On Weyl's theorem for quasi-class A operators, J. Korean Math. Soc., 43(2006), 899-909.

[7] T. Furuta, M. Ito and T. Yamazaki, A subclass of paranormal operators including class of log-hyponormal and several related classes, Sci. Math., 1(1998), 389-403.

[8] Halmos P. R., A Hilbert Space Problem Book, Second Edition, Springer-Verlag, New York 1982. 
[9] M. Ito, Several properties on class A including p-hyponormal and log-hyponormal operators, Math. Ineq. Appl., 2(1999), 569-578.

[10] I. H. Jeon, J. I. Lee and A. Uchiyama, On p-quasihyponormal operators and quasisimilarity, Math. Ineq. App., 6(2003) 309-315.

[11] I. H. Jeon and I. H. Kim, On operators satisfying $T^{*}\left|T^{2}\right| T \geq T^{*}|T|^{2} T^{*}$, Linear alg. Appl., 418(2006), 854-862.

[12] I. H. Kim, On ( $p, k)$-quasihyponormal operators, Math. Ineq. Appl., 4(2004), 169-178.

[13] A. Uchiyama, Weyl's theorem for class A operators, Math. Ineq. App., 4(2001), 143-150.

Department of Mathematics and Statistics, Faculty of Science P.O. Box(7), Mu'tah University, Mu'tah-Jordan.

E-mail: malik_okasha@yahoo.com

School of Mathematical Sciences, Faculty of Science and Technology, Universiti Kebangsaan Malaysia, 43600 UKM, Selangor Darul Ehsan, Malsysia.

E-mail: msn@pkrisc.cc.ukm.my 